\title{
Trauma de la pared torácica y manejo del neumotórax en equinos: reporte de un caso clínico atendido bajo condiciones de campo.
}

\section{Equine thoracic wall trauma and management of pneumothorax: report of a clinical case attended under field conditions}

\begin{abstract}
Juan Estrada M1区 (jmevet@hotmail.com),Javier Montero U1(javmontero56@hotmail.com), José Vargas $A^{1}$ (josevargas29@gmail.com), Manuel Estrada U1'(mestradavet@yahoo.com),
\end{abstract}

1 Hospital Equino, Escuela de Medicina Veterinaria, Universidad Nacional, Costa Rica.

Recibido: 4 de Octubre de 2013. Corregido: 8 de Enero de 2016. Aceptado: 15 de Enero de 2016.

\begin{abstract}
Various causes of accidental trauma affecting the equine thoraxic wall and the pathophisiology of pneumothorax are mentioned. Diagnosis techniques, as well as medical and surgical management of the clinical case on a standing horse under field conditions are discussed. Two options used to depressurize the pleural cavity are explained. The paper also mentions the advantages of handling the case under local anesthetic techniques versus general anesthesia. All the procedures were performed at a farm located in the canton of Coronado, Costa Rica.
\end{abstract}

Keywords: thoracic trauma, pneumothorax, standing horse, field conditions, depressurize, pleural cavity, Costa Rica

Resumen: Se menciona varias causas de trauma accidental que afectan la pared torácica equina y la patofisiología del neumotórax. Se discuten técnicas para el diagnóstico; manejo médico y quirúrgico del caso, con el caballo en pie, bajo condiciones de campo. Se explican dos opciones utilizadas para despresurizar la cavidad pleural. Se plantean las ventajas de manejar el caso con bloqueo anestésico local versus anestesia general inhalatoria. Los procedimientos se efectuaron en una finca ubicada en el Cantón de Coronado, Costa Rica.

Palabras clave: trauma torácico, neumotórax, caballo en pie, condiciones de campo, despresurizar, cavidad pleural, Costa Rica.

\section{INTRODUCCIÓN}

El tórax es una cavidad compleja desarrollada, evolutivamente, para maximizar la eficiencia respiratoria. El trauma torácico, con sus complicaciones asociadas, tales como fallo pulmonar y cardiaco, lesión de vasos sanguíneos, fracturas de huesos adyacentes, laceración de vísceras abdominales, infección, endotoxemia y alteraciones de la presión negativa pleural, pueden amenazar la vida del animal afectado (Sprayberry \& Barrett 2015), "El veterinario

$\triangle$ Autor de correspondencia:

jmevet@hotmail.com 
debe estar preparado para atenderlo" (Peroni 2011). El esqueleto del tórax comprende 18 vértebras, ubicadas dorsalmente; 18 pares de costillas y el esternón, ventralmente; todo cubierto por músculos, fascias y piel, lo cual forma una caja protectora para los órganos internos (Getty 1975; Clayton et al. 2007; Jeffcott 2009). Los pulmones están localizados dentro de la cavidad pleural, que es un espacio cubierto por membrana pleural (parietal y visceral) el cual yace dentro de la cavidad torácica. Esta cavidad pleural se dividida en dos cavidades (hemitórax izquierdo y derecho) por un septo de tejido conectivo, llamado mediastino; el cual, a su vez, contiene al corazón, los grandes vasos sanguíneos, nervios, tejido adiposo; además, segmentos de la tráquea y el esófago. Los equinos tienen, comúnmente, el mediastino incompleto, debido a fenestraciones que producen una comunicación funcional entre las dos cavidades pleurales (Budras et al. 2009). La inhalación (inspiración) ocurre cuando la contracción del diafragma y los músculos intercostales externos expanden el tórax, creando una presión intra-alveolar negativa ( $1 \mathrm{~mm} \mathrm{Hg}$ más baja que la atmosférica), la cual induce un flujo pasivo de aire de la atmósfera hacia el alveolo. La exhalación (espiración) es pasiva, como resultado del aire que sale de los pulmones cuando estos se retraen a su dimensión original. Si todo funciona normal, el tórax y los pulmones ciclan rítmicamente a través de fases de expansión y retracción; el aire entra y sale del alveolo y el intercambio gaseoso ocurre en la interfase existente entre los capilares pulmonares y el alveolo (Sprayberry \& Barrett 2015). En la cavidad pleural, la presión constante es siempre de unos $4 \mathrm{mmHg}$ menor ("más negativa") que la presión alveolar (aire atmosférico), por lo cual, este vacío permite que los pulmones se mantengan en un estado de expansión parcial y llenen una cavidad torácica más grande que el pulmón durante todas las fases del ciclo respiratorio, incluyendo espiración. Esta presión negativa se mantiene constante porque el espacio pleural es cerrado y no se comunica con el alveolo o la atmósfera, previniendo el colapso pulmonar, incluso durante la espiración forzada. Los movimientos respiratorios normales, de la pared toracal y el pulmón, tienden a separar ambas estructuras, pero la existencia de un fluido seroso dentro de la cavidad pleural tiene un efecto cohesivo que contrarresta dicha separación al mantener juntas las superficies pleurales parietal y visceral, permitiendo que los pulmones estén perfectamente acoplados a la cavidad torácica y sigan sus movimientos. Por estas razones, la cavidad pleural, del animal sano, debe entenderse como un espacio potencial más que como una cavidad (Sprayberry \& Barrett 2015). Debido a la amplitud del tema, otros aspectos de la fisiología de la pared torácica, cavidad pleural, pulmones y respiración, no se incluyen en este artículo, los cuales son discutidos ampliamente en varias publicaciones (Left et al. 1993; McDonell et al. 2007; Robinson 2007; Sprayberry \& Barrett 2015). Dicha presión negativa de la cavidad pleural mantiene los pulmones expandidos; pero, cuando la caja torácica es perforada, el aire atmosférico entra al espacio pleural, generando una presión positiva, la cual desinfla el pulmón del lado respectivo y causa su colapso. Los caballos tienen el mediastino incompleto; de ahí que la lesión de un hemitórax afecta al hemitórax contralateral, causando neumotórax bilateral (Sprayberry \& Barrett 2015). El neumotórax equino tiene una patofisiología multifactorial, pues involucra aspectos anatómicos, fisiológicos e infecciosos, siendo el trauma la principal causa del problema (Hassel 2007; Peroni 2011; Trigo et al. 2011; Sprayberry \& 
Barrett 2015). Entre las principales causas, que afectan la integridad anatómica de la pared torácica equina, se encuentran las heridas punzantes, colisión con objetos, heridas por bala, golpes (Ej. Patadas), fracturas costales y laceraciones causadas por cornadas. Fractura de costillas son más comunes en potrillos (Knottenbelt et al. 2004; Hassel 2007; Trigo et al. 2011) que en adultos, ocurren durante el parto o por trauma directo, pues los neonatos pasan mucho tiempo acostados (Laverty et al. 1999; Bellezzo et al. 2004; Schambourg et al. 2004) y pueden ser pisoteados por la madre. El manejo del trauma torácico requiere, como prioridades: 1) asegurar una vía aérea, 2) tratar el shock, 3) instaurar profilaxis contra tétano y 4) hacer control de infección por contaminantes ingresados a la pleura (Hassel 2007). Las heridas axilares profundas bombean aire hacia adentro del cuerpo cuando el paciente se mueve, causando enfisema subcutáneo que puede migrar y producir neumomediastino y/o neumotórax (Hance et al. 1992; Lugo 2006) por lo que, cualquier insuficiencia respiratoria debe monitorearse en pacientes con este tipo de heridas. Trauma torácico (Figura 1), especialmente caudal a la sexta costilla (Boy et al. 2000; Lugo 2006) podría causar trauma abdominal; se le debe prestar especial atención (Sprayberry \& Barrett 2015).

El neumotórax causa hipoxemia por distribución irregular del aire inspirado (Maud-Aline et
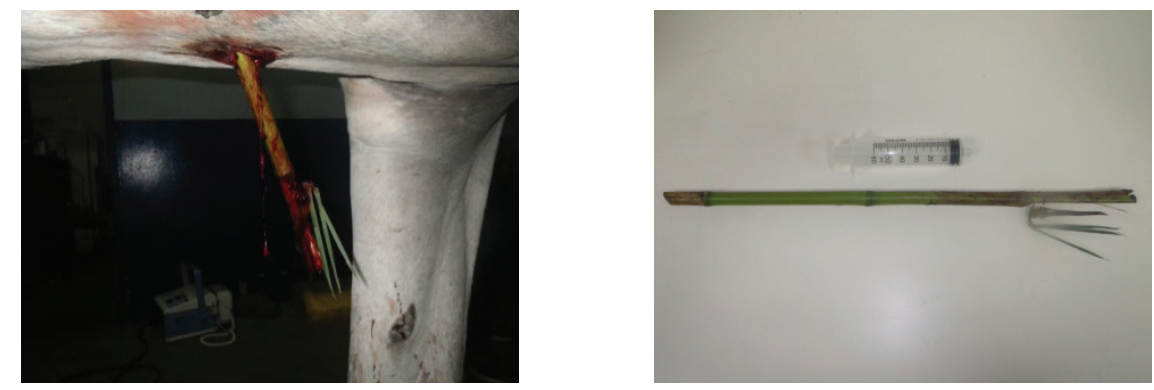

Figura 1. Laceración profunda causada por una estaca de bambú que penetró la pared torácica y el abdomen causando neumotórax, ruptura visceral y peritonitis (Estrada M., datos no publicados).

at. 2012). El diagnóstico de neumotórax se hace basado en signos clínicos, principalmente la marcada insuficiencia respiratoria y el ruido efectuado por el aire que sale de la herida. El examen clínico incluye una inspección visual para obtener una perspectiva global del paciente. Observar el patrón respiratorio del paciente, idealmente, desde un punto ubicado encima del animal, puede revelar asimetría respiratoria entre lados izquierdo y derecho del tórax, deformación ósea local, rigidez y/o movimiento paradójico del tórax ("flail chest") como discutido luego (ver Figura 8). Un caballo que tenga una combinación de taquipnea, disnea, elevación de ollares, actitud inquieta o ansiosa, membranas mucosas pálidas o cianóticas, debe ser categorizado como sospechoso por neumotórax. Se debe hacer una palpación digital exhaustiva del área, alrededor de la herida, para detectar enfisema subcutáneo y crépito. Matidez o ausencia de sonidos pulmonares ventrales puede sugerir hemotórax o efusión pleural, mientras que ausencia de sonidos pulmonares dorsales, en 
el caballo de pie, puede indicar neumotórax (Sprayberry \& Barrett 2015). Las imágenes diagnósticas deben considerarse parte del examen completo inicial, pues son indicadas para monitorear la evolución del caso, incluyendo complicaciones como hemotórax o neumotórax (Sprayberry \& Barrett 2015). El uso de ultrasonido (Reef et al. 1991) y los rayos X (Butler et al. 2003) confirman las sospechas clínicas. Cuando el pulmón se infla y desinfla durante la respiración, ocurre, normalmente, un movimiento deslizante ("glide sign") por contacto entre las pleuras visceral y parietal, el cual se determina ultrasonográficamente (Sprayberry \& Barrett 2015); además de un artefacto de "cola de cometa." Ambos signos ecográficos desaparecen en casos de neumotórax, puesto que la pleura visceral colapsa junto con el pulmón respectivo, perdiéndose el contacto con la pleura parietal. En su defecto, aparecen líneas horizontales paralelas de reverberación por aire, sin el patrón de pleuras deslizantes ni los artefactos de "cola de cometa" (Axon 2010). Para la ultrasonografía torácica equina, los mismos autores recomiendan el uso de transductores curvos con rango de 2.5 a 5.0 $\mathrm{MHz}$. Imágenes radiográficas normales del tórax equino son descritas en la literatura (Butler et al. 2003; Weaver \& Barakzai 2009). Las radiografías latero-mediales del tórax, en caballos con neumotórax bilateral, muestran una imagen radiolucente por ausencia de la vascularización pulmonar normal en la región dorsal de los campos pulmonares caudales y desplazamiento ventral del margen pulmonar dorsal (Axon 2010). Según Butler et al. (2003), en neumotórax, "el aire en el espacio pleural rápidamente se acumula dorsalmente, en el receso paraespinal y en la reflexión caudal dorsal de la pleura adyacente al diafragma en el punto más alto de dicho receso paraespinal. Por tanto, el área dorsocaudal del tórax es la zona más importante de evaluar cuando se esperan pequeñas cantidades de gas. Radiográficamente, los márgenes pulmonares están retraídos caudalmente del diafragma y dorsalmente de los cuerpos vertebrales, al permitir la visualización de la superficie pleural del pulmón. Neumotórax unilateral puede ocurrir y, en estos casos, es posible ver el margen de un pulmón retraído y visualizar los vasos sanguíneos del pulmón contralateral. En casos de neumotórax bilateral, ambos lóbulos pulmonares están retraídos. El tórax completo debe revisarse por otros signos de patología tales como costillas fracturadas o cuerpos extraños". Una nueva radiografía, tomada luego de la resolución del neumotórax, muestra el retorno a la vascularización normal (Axon 2010). La toracoscopía (Peroni et al. 2001; Lugo 2006; Sprayberry \& Barrett 2015), muestra el pulmón colapsado. Medir los gases arteriales cuantifica la severidad de la disfunción (Maud-Aline et al. 2012) y el hemograma monitorea leucocitosis. El manejo de un neumotórax abierto demanda la oclusión de la herida, como primera línea de terapia, para prevenir más entrada de aire y contaminantes a la cavidad pleural (Sprayberry \& Barrett 2015). Lo anterior, se logra usando un sello a prueba de aire (Ej. lobán®, 3M) o suturando el defecto (Sprayberry \& Barrett 2015), con técnicas quirúrgicas rutinarias a gusto de cada cirujano, para cerrar los diferentes planos tisulares de la herida, tales como patrón simple continuo o interrumpido. Se recomienda, (Laverty et al. 1996; Boy et al. 2000; Lugo 2006) al menos, empacar la herida, mientras se puede suturar, para bajar la contaminación pleural e inyectar antibiótico de amplio espectro, incluyendo acción contra gérmenes anaeróbicos y antitoxina tetánica. En lesiones infectadas, se deben seguir las recomendaciones del antibiograma. Implementar un protocolo analgésico es 
mandatorio, pues, además de las razones éticas del caso, el dolor causado por el trauma limita los movimientos respiratorios y la ventilación (Lugo 2006), por lo que se recomiendan AINE'S y/o anestesia intercostal con infusión profunda. Anestesia general se indica (Lugo 2006) en heridas extensas, lesiones con fracturas costales o animales de manejo difícil, estabilizando previamente el paciente o incluso posponiendo la cirugía por 2-3 días. MaudAline et al. (2012) describen el manejo de neumotórax bajo anestesia general. La cirugía siempre se justifica en neumotórax con herida penetrante. Una vez suturado o empacado el defecto, sigue el tratamiento médico y la estabilización metabólica (fluidos, esteroides, oxigenoterapia). Se recomienda suplementar oxígeno con un flujo inicial de $15 \mathrm{~L}$ por minuto (Sprayberry \& Barrett 2015). Al combinar varias técnicas, reportadas por la literatura (Stone et al. 1994; Laverty et al. 1996; Laverty et al. 1999; Boy et al. 2000; Bellezzo et al. 2004; Schambourg et al. 2004; Hendrickson 2006; Lugo 2006; Hassel 2007; Sprayberry \& Barrett 2015), el procedimiento para la estabilización del paciente se puede efectuar bajo anestesia, local con el animal de pie. Luego de cerrada la herida, la cavidad pleural se despresuriza haciendo una punción con un instrumento tubular hueco ("cánula") que atraviesa la pared torácica y se acopla a una llave de triple paso, a través de la cual el aire intrapleural se extrae usando jeringa o aparato de succión (Sprayberry \& Barrett 2015); siendo, además, el "sello de agua" una opción utilizada por décadas en la medicina mundial. Hardy (2004) recomienda usar el doceavo espacio intercostal para efectuar la punción, mientras que Sprayberry \& Barrett (2015) mencionan el aspecto dorsal de los espacios 12 a 15.

\section{MATERIALES Y MÉTODOS}

\section{Anamnesis}

Una yegua angloárabe, 8 años de edad, gestante, fue atendida en la finca de residencia unas 12 horas post accidente. Los encargados reportan una pequeña herida sobre el costado derecho (ver Figura 2), insuficiencia respiratoria al caminar y un ruido particular producido por el aire que sale del tórax a través de la herida, el cual es acompañado con espuma sanguinolenta. El veterinario tratante indicó, vía telefónica, cubrir la herida con un vendaje provisional, iniciar terapia con antibióticos e inyectar meglumina de flunixín, mientras el animal era atendido en forma definitiva.

\section{Diagnóstico}

El diagnóstico se basó en historia y signos clínicos. El examen general mostró, además, tos y mocos moderados, disminución de ruidos respiratorios por auscultación, intolerancia al ejercicio, mucosas cianóticas y enfisema subcutáneo perilesional, más la herida toracal. 


\section{Tratamiento médico preoperatorio}

En el lugar del accidente, la herida se limpió y empacó con gasa empapada en Clorexidina $0,2 \%$, recubriéndose con un vendaje para bajar la contaminación. Se inyectó antibiótico profiláctico y antitoxina tetánica. Posteriormente, el paciente fue trasladado, caminando lentamente, por unos 500 metros hasta un sitio menos contaminado, en el cual se efectuó la sutura y despresurización de la cavidad pleural.

\section{Anestesia y Cirugía}

La operación se efectuó con el paciente de pie, inmovilizado en un cepo rústico (ver Figura 2). El sitio quirúrgico se depiló en forma adecuada y, posteriormente, se desinfectó con jabón de clorhexidina más alcohol-yodo. Se anestesió localmente con Lidocaína $2 \%$ (Laboratorios Faryvet S.A, Costa Rica) en patrón de "L" invertida. Se introdujo, brevemente, un instrumento estéril de punta roma (Ej. Tijera o pinza hemostática) en la herida (Figura 2) para determinar la dirección y profundidad, tapando de inmediato el defecto para prevenir más contaminación intrapleural y entrada de aire atmosférico mientras se sutura.
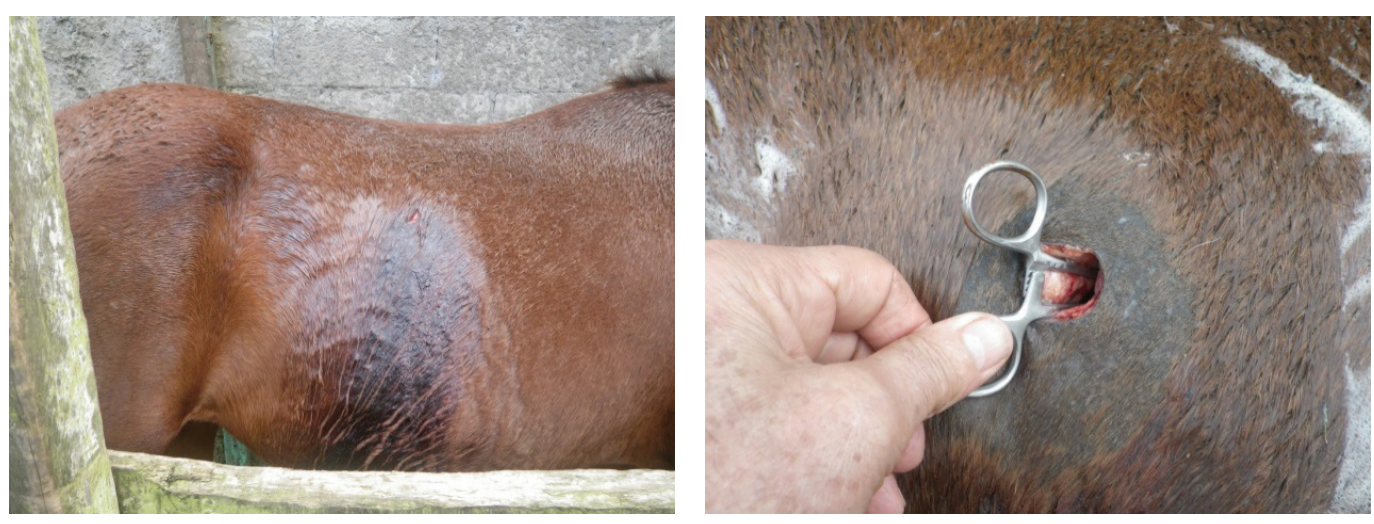

Figura 2. Se observa la pequeña herida, ocurrida unas 12 horas antes. Muestra salida de espuma sanguinolenta durante espiración. El instrumento atraviesa el espacio intercostal (foto derecha) y apunta hacia cráneo-ventral invadiendo el hemitórax respectivo.

Los planos internos, incluyendo subcutáneo, se suturaron en dos capas con Dexón ${ }^{R}$ No. 0 en patrón "simple continuo", para sellar herméticamente la entrada o salida del aire a la cavidad pleural, pudiendo usarse un pedículo muscular para mejorar el sello (Stone et al. 1994). La piel fue suturada con patrón "simple interrumpido" usando Nylon 0. Finalmente, se usó un patrón en "colchonero horizontal" como punto de retención para reforzar el cierre hermético citado (ver Figura 4). Una vez suturada la herida, se sacó el aire pleural (despresurización) siguiendo el procedimiento indicado en las Figuras 4 a 7. 


\section{Técnica usada para despresurizar el tórax}

El equipo usado para despresurizar el tórax consistió (Figura 3) en: 1) cánula metálica con punta roma, 2) aguja larga de grueso calibre, 3) llave de triple paso, 4) equipo infusor desechable y 5) bolsa o botella de fluidos para uso intravenoso.
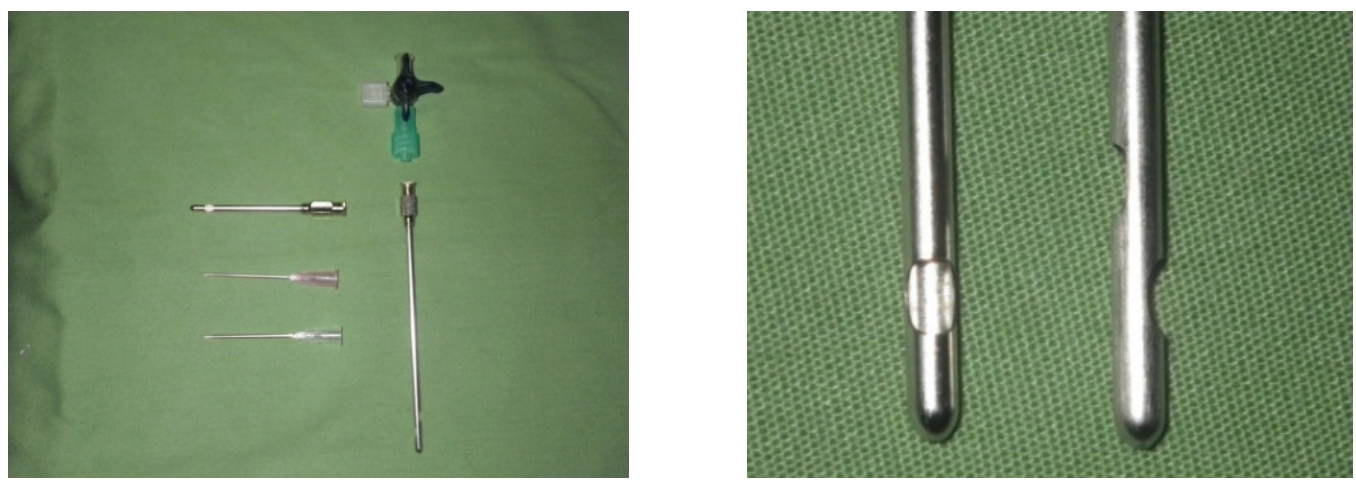

Figura 3. Se muestra la llave de triple paso más varios tipos de cánulas o las agujas usadas para sacar aire de la cavidad pleural. A la derecha se ve la punta roma y los agujeros de irrigación de la cánula metálica.

En este caso, para despresurizar, se hizo punción con una cánula roma que atravesó la pared torácica comunicando la cavidad pleural con el ambiente exterior. Para poner la cánula, el punto escogido fue depilado y desinfectado; se infiltró localmente con $3 \mathrm{ml}$ de Lidocaína $y$, unos 5 minutos después, se hizo una pequeña incisión con un bisturí para introducir la cánula hasta la pleura. La cánula se fijó a piel con pegamento instantáneo de cianocrilato (Loctite Super Bonder®, Henkel Ltd) más un punto de sutura, para que dicha cánula no se desacople de la piel cuando se efectúa la succión del aire torácico o por movimientos del paciente. Inmediatamente, la llave de triple paso cerrada fue acoplada a la cánula (Figura 4). A partir de este momento el aire pleural se puede sacar por succión con jeringa o usando un "sello de agua".

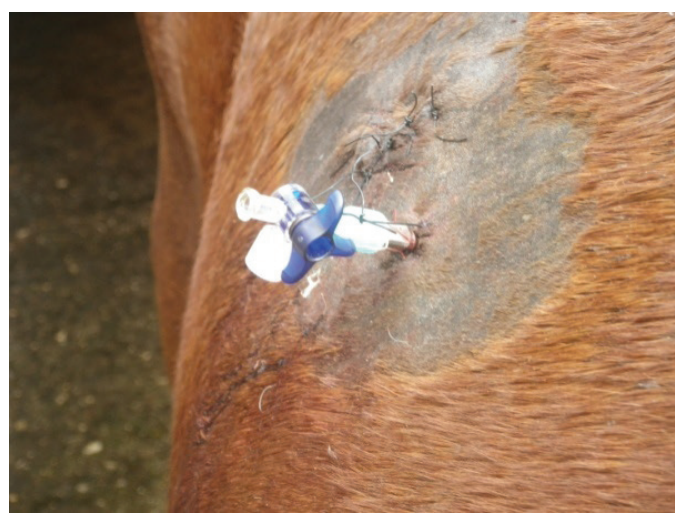

Figura 4. La llave de triple paso cerrada se acopló a la cánula mamaria y se fijó a la piel, pudiendo usarse jeringa o "sello de agua" para sacar el aire pleural. 
En el primer caso, una jeringa de $60 \mathrm{ml}$ se acopló a la llave de triple paso (Figura 5) para succionar aire pleural hasta llenarla.
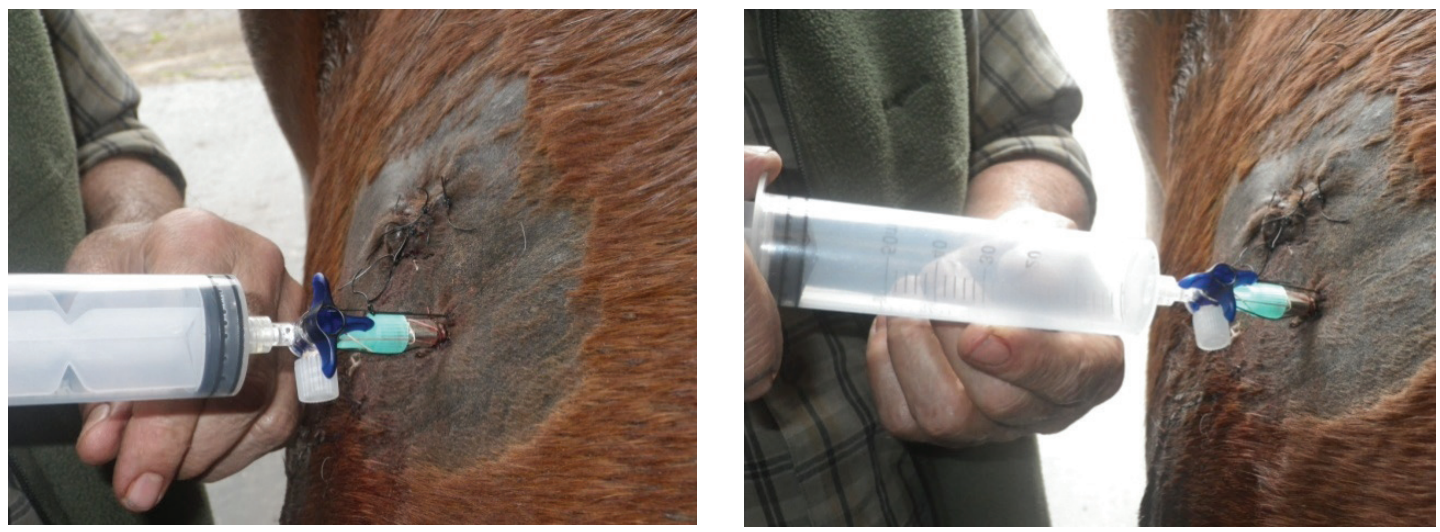

Figura 5. Se aprecian la jeringa y llave de triple paso acopladas. Abriendo la llave (foto izquierda) el aire intrapleural fue succionado con la jeringa. Antes del desacople se debe cerrar la llave (foto derecha).

Luego se cerró la llave, se desacopló la jeringa y el aire succionado fue eliminado. (Figura 6) Se repitió el ciclo unas 100 veces hasta sacar todo el aire pleural posible; en este momento el paciente comenzó a normalizar la respiración. Si fuera necesario, se puede continuar el procedimiento por varios ciclos más.
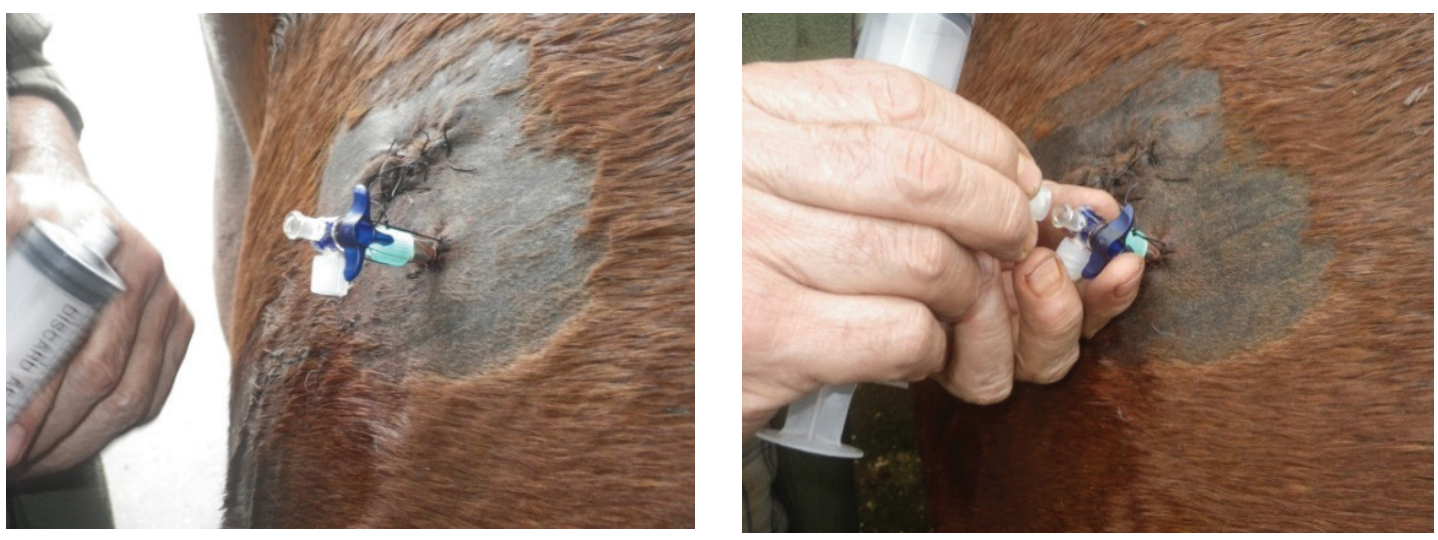

Figura 6. Con la llave cerrada, el aire succionado se eliminó de la jeringa. La llave (lado derecho) se tapa entre succiones para bajar contaminación. 
Cuando se usa un "Sello de agua" el aire del espacio pleural sale por la cánula, sigue por el tubo de infusión y llega hasta el agua del recipiente, donde produce burbujas que flotan por gravedad y salen a la atmósfera. El aire atmosférico no puede viajar en sentido contrario (del exterior del animal hasta la cavidad pleural) porque el agua forma una "válvula de una vía" que lo impide. Así, el aire pleural sale durante espiración y el aire atmosférico no entra a cavidad pleural durante inhalación. Es importante recordar: 1) el líquido debería ser estéril, 2) se debe mantener el recipiente en posición vertical debajo del pulmón para que el líquido no retorne al tórax y 3) reponer el líquido evaporado. Si se usa una bolsa de fluidos fisiológicos, como sello de agua, entonces el aire pleural, vía tubo de infusión, entra al líquido por debajo de la bolsa formando burbujas que flotan hacia arriba (monitorear) y salen a la atmósfera por una aguja insertada o un piquete hecho en la parte superior de la bolsa. Esta bolsa se puede colgar de un clavo o fijar al paciente (monitorear). Para hacer el "sello," usando una botella, (Figura 7) es necesario que el recipiente tenga un tapón con dos agujeros, uno para que el aire proveniente del tórax pase al líquido y el segundo para sacar aire desde la botella hasta la atmósfera.
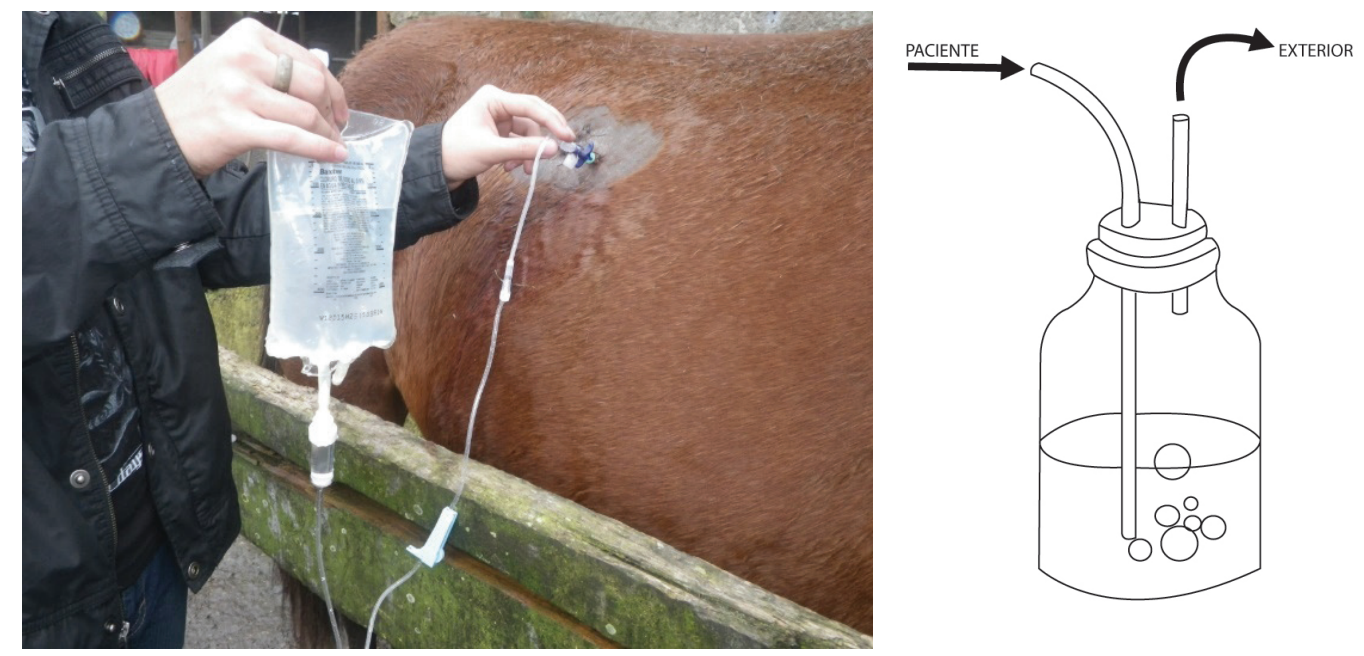

Figura 7. La fotografía muestra una bolsa de fluidos estériles que fue usada como sello de agua para tratar el caso clínico descrito. La bolsa debe mantenerse más abajo que la cánula para que el líquido no pase al tórax mientras que permite al aire pleural salir. Como medida de seguridad, es fácil observar si están saliendo burbujas hacia arriba del líquido (correcto) o si el fluido del recipiente está corriendo por el tubo plástico hacia el tórax (incorrecto). En el dibujo del lado derecho se ilustra el mismo principio usando una botella para contener el fluido. 


\section{Postoperatorio}

El procedimiento postoperatorio incluyó examen clínico general cada 48 horas, / 3 veces, para monitorear infección (respiratoria, herida o punción) más observación de parámetros como: curva de temperatura, apetito, actitud, tos y secreciones mucosas nasales por 6 días; hemograma de control al final del tratamiento; antibióticos y AINE'S sistémicos por 6 días; lavados locales por 10 días y remoción de suturas al final de este período.

\section{RESULTADOS Y DISCUSIÓN}

Los principios generales aquí discutidos, para el manejo del neumotórax, se pueden aplicar en otras especies animales. Para el cirujano es más fácil trabajar en un hospital que en la finca, incluyendo el control de la contaminación, pero nuestra experiencia demuestra que se puede manejar la mayoría de los casos de neumotórax, con el paciente de pie, bajo condiciones de campo y sin necesidad de transportarlo inestable por largas distancias, lo cual es ventajoso porque disminuye los costos y "evita complicaciones respiratorias y cardiovasculares que pueden ocurrir durante la anestesia general," (Lugo 2006), al mejorar el pronóstico clínico y lograr el retorno del paciente a su función original en menor tiempo. Los autores prefieren no usar sedativos durante el procedimiento quirúrgico, pues consideran que, como el déficit respiratorio deprime al paciente, este generalmente no muestra reacciones violentas ante estímulos, incluyendo cirugía. Peroni (2011) menciona que "cuando los veterinarios evalúan caballos con lesiones de tórax pueden enfrentar el reto del diagnóstico y manejo de un neumotórax. Heridas torácicas abiertas presentan retos relacionados con la infección pleural, cuerpos extraños y fracturas de costillas". Cuando una costilla se fractura en dos sitios, el segmento de costilla entre ambas líneas de fractura queda mecánicamente aislado de los segmentos proximal y distal. Cuando dos o más costillas contiguas se fracturan en dos o más sitios se forma una "isla" de pared torácica, la cual está desacoplada funcionalmente de la pared toracal, pues el segmento aislado se mueve en dirección contraria a la pared normal durante inspiración y espiración; este fenómeno es conocido como movimiento paradójico de la pared torácica o "flail chest" en idioma Inglés (Sprayberry \& Barrett 2015). Una costilla fracturada puede lacerar un pulmón causando neumotórax; pero, según la experiencia de los autores, algunas fracturas costales (Figura 8) no siempre causan neumotórax, lo cual implica un diagnóstico diferencial.

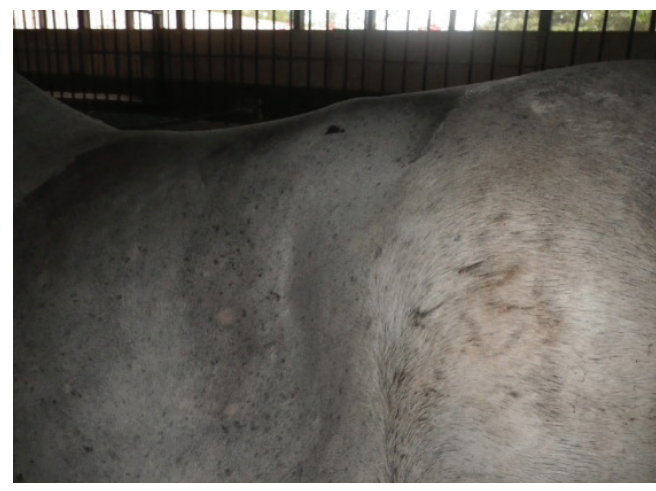

Figura 8. Paciente mostrando un área de depresión aislada en el tórax izquierdo correspondiente a la fractura por trauma de varias costillas caudales contiguas las cuales muestran movimiento paradójico sin neumotórax concurrente. 
Respecto a la escogencia del sitio de la punción transtoracal con la cánula roma, en la experiencia de los autores, cualquier espacio intercostal sirve para el procedimiento, en tanto esté cercano a la herida y el área no presente signos de trauma o enfisema subcutáneo que predisponen a infección local. El sitio de la punción debe ubicarse a la misma altura o dorsalmente a la herida para que la cánula no cause trauma al pulmón conforme este se expande como resultado de la terapia; además, usar una cánula de punta roma disminuye este riesgo. Un oxímetro de pulso portátil, (Ej. Pulse Oximeter, Modelo PM-60 Vet, Mindray Inc, China) aplicado en la lengua, ayuda a cuantificar el déficit respiratorio, pues si la lectura normal es de 95-100\% entonces, valores debajo de esta cifra sugieren la severidad de la hipoxia., lo cual permite, al clínico, tomar decisiones tales como suplementar con 02 (si está disponible en el sitio) o intentar movilizar un paciente inestable del sitio del accidente hasta un lugar más adecuado, incluso a través de terreno difícil, para instaurar el tratamiento.

En el caso descrito, la yegua no presentó secuelas del accidente; tuvo parto normal, seis meses post-cirugía y se continúa montando luego del destete.

\section{RECOMENDACIONES}

Es importante eliminar del ambiente objetos potencialmente traumáticos para animales o personal. Se debe educar a los propietarios y encargados del caballo para que, ante la ocurrencia de heridas torácicas accidentales, llamen de inmediato al Médico Veterinario para que categorice y atienda la emergencia.

\section{AGRADECIMIENTOS}

Nuestro reconocimiento a los encargados del animal, Sr. Jesús Chacón y Sra. Marianela Porras, Coronado, Costa Rica, por su invaluable ayuda.

\section{REFERENCIAS}

Axon, J.E. 2010. Traumatismo torácico. In B.P. Smith (ed). Medicina Interna de Grandes Animales, Elsevier, Barcelona. p. 551-553.

Bellezzo, F., Hunt, R.J., Probost, R., Bain, F.T. \& Kirker-Head, C. 2004. Surgical repair of rib fractures in 14 neonatal foals: case selection, surgical technique and results. Equine Vet J.36:557.

Boy, M.G. \& Sweeney, C.R. 2000. Pneumothorax in horses: 40 cases (1980-1997). J. Am Vet Med Assoc. 216:1955-1959.

Budras, K., Sack, W.O. \& Rock, S. 2009. Thoracic cavity. In Budras, K., Sack, W.O., Rock, S., (eds.), Anatomy of the horse, $5^{\text {th }}$ edition. Hannover (Germany): Schlutersche Verlagsgesellschaft mbH \& Co KG. p.60.

Butler, J.A., Colles, C.M., Dyson, S.J., Kold, S.E. \& Poulos, P.W. 2003. Clinical Radiology of the Horse, Second Edition, Blackwell Science, Oxford. p. 485, 486-503. 
Clayton, H.M., Flood, P.F. \& Rosenstein, D.S. 2007. Anatomía Clínica del Caballo. Elsevier, Amsterdam. p. 89-96.

Getty, R. 1975. Sisson and Grossman's The Anatomy of Domestic Animals. $5^{\text {th }}$ Edition. WB Saunders, Philadelphia.

Hance, S.R. \& Robertson, J.T. 1992. Subcutaneous emphysema from an axillary wound that resulted in pneumomediastinum and bilateral pneumothorax in a horse. J Am Vet Med Assoc. 200:1107.

Hardy, J. 2004. Emergency Procedures and First Aid. p. 1185. In K.W. Hinchcliff, A.J. Kaneps \& R.J. Geor, (eds.). Equine Sports Medicine and Surgery. Saunders, Edinburgh.

Hassel, D.M. 2007. Thoracic Trauma in Horses. Veterinary Clinics of North America: Equine Practice. 23(Issue 1): 67-80.

Hendrickson, D.A. 2006. Management of Deep and Chronic Wounds. p. 299-305. In J.A. Auer \& J.A. Stick, (eds.). Equine Surgery. $3^{\text {rd }}$ Edition. Saunders Elsevier, St Louis Missouri.

Jeffcott, L.B. 2009.The Normal Anatomy of the Osseus Structures of the Back and Pelvis. p. 3-15. In F.M.D. Henson, (ed.). Equine Back Pathology Diagnosis and Treatment. WileyBlackwell, Oxford.

Knottenbelt, D.C., Holdstock, N. \& Madigan, J.E. 2004. The foal at the delivery. p. 65-74. In D.C. Knottenbelt, (ed.). Equine Neonatology: Medicine and Surgery. Saunders, Philadelphia.

Laverty, S., Lavoie, J.P., Pascoe, J.R. \& Ducharme, N. 1996. Penetrating wounds of the thorax in 15 horses. Equine Vet J. 28:220-224.

Laverty, J.D. \& Halley, J. 1999. Thoracic trauma in newborn foals. Equine Vet J. 31:149.

Left, A.R. \& Schumacher, P.T. 1993. Respiratory Physiology: Basics and Applications. WB Saunders, Philadelphia.

Lugo, J. 2006. Thoracic Disorders. p. 616-623. In J.A. Auer \& J.A. Stick, (eds.). Equine Surgery.

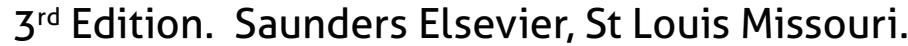

Maud-Aline, C., Aprea, F. \& Clutton, E. 2012 June. Anesthetic management of a horse with traumatic pneumothorax. Can Vet J. 53(6):648-652.

McDonell, W.N. \& Kerr, C.L. 2007. Respiratory System. p. 117-151. In Tranquilli, W.J., Thurmon, J.C. \& Grimm, K.A. (eds.). Lumb \& Jones Veterinary Anesthesia and Analgesia. $4^{\text {th }}$ Edition. Blackwell Publishing, lowa.

Peroni, J.F., Horner, N.T., Robinson N.E. \& Stick, J.A. 2001. Equine thoracoscopy: normal anatomy and surgical technique. Eq Vet J. 33 (3):231-237.

Reef, V.B., Boy, M.G., Reid, C.F. \& Esler, A. 1991. Comparison between diagnostic ultrasonography and radiography in the evaluation of horses and cattle with thoracic disease: 56 cases (1984-1985). J Am Vet Med Assoc. 198:2112. 
Robinson, N.E. 2007. How horses breathe: The respiratory muscles and the airways. p.19-31. In McGorum, B.C., Dixon, P.M., Robinson N.E. \& Schumacher, J. (eds.). Equine Respiratory Medicine and Surgery. Saunders Elsevier, Philadelphia.

Schambourg, M.A., Laverty, S. \& Mullim, S. 2004. Thoracic trauma in foals: Postmortem findings. Equine Vet J.35:78.

Sprayberry, K.A. \& Barrett, E.J. 2015. Thoracic trauma in horses. Vet Clin Equine 31: 199-219 or Vet Clin Equine 31 (2015) 199-210, Según DOI

Stone, W.C., Trostle, S.S. \& Gerros, T.C.1994. Use of a primary muscle pedicle flap to repair a caudal thoracic wound in a horse. J Am Vet Med Assoc. 205:828.

Trigo, P., Muñoz, A., Castejón, F., Riber C. \& Hassel, D.M. 2011 (Nov). Rib fracture in a horse during an endurance race. Can Vet J. 52(11):1226-1227.

Weaver, M. \& Barakzai, S. 2009. Radiographs of the thorax. p.161-167. In Handbook of Equine Radiography, Saunders Elsevier, U.K. 